\title{
Intervención en poblaciones de riesgo (2): los bebedores de riesgo
}

\author{
Colom, J.; Contel, M.; Segura, L.; Gual, A. \\ Dirección General de Drogodependencias y Sida. Departamento de Sanidad y Seguridad Social. Generalitat de Catalunya
}

Enviar correspondencia a: Montserrat Contel. Trav. Les Corts, 131-159. Pavelló Ave Maria. 08028 Barcelona. Tel. +34 935566352.

Fax. +34 $932272974 \cdot$ E-mail: mcontel@dsss.scs.es

\section{RESUMEN}

La mayor parte de daños y costes sociosanitarios asociados al alcohol se producen en sujetos consumidores no dependientes. El 9,5\% de la población adulta española son bebedores de riesgo. La OMS, mediante el Collaborative Study on Alcohol Early Interventions in Primary Health Care Settings, ha desarrollado una estrategia de detección e intervención precoz en bebedores de riesgo. La primera fase del estudio permitió validar un instrumento de cribado (el cuestionario AUDIT). En la segunda fase se evidenció la efectividad de las intervenciones breves (Drinkless program), y en la tercera se analizaron cuales son las estrategias de marketing y formación para diseminar las intervenciones breves en la atención primaria.

La cuarta fase se centra precisamente en la diseminación generalizada de las intervenciones breves en atención primaria. El programa 'Beveu Menys' (Beba menos) es la experiencia pionera. Mediante técnicas de formación de formadores y creando una intensa coordinación entre la atención primaria y los centros de drogodependencias, se ha desarrollado un programa que permite abordar tanto a los consumidores de riesgo como a los pacientes con dependencia alcohólica.

Palabras clave: Atención primaria de salud, intervención breve, bebedor de riesgo, alcoholismo, reducción del daño.

\section{ABSTRACT}

Most of the alcohol related harm occurs in non dependent drinkers. 9.5\% of spanish adult population are risky drinkers. WHO conducts through the WHO Collaborative Study on Alcohol Early Interventions in Primary Health Care Settings, a cross-national study which aims to develop effective instruments for early detection and brief intervention in risky drinkers. Phase I of the Study was devoted to the design and validation of a screening tool (the AUDIT questionnaire). In Phase II, efficacy of brief interventions (using the Drinkless program) was shown. Phase III focused on marketing and support strategies most efficient to widespread brief interventions in Primary Health Care.

Phase IV aims at real dissemination of brief interventions in Primary Health Care. The 'Beveu Menys' (Drink less) program is pioneering the experience. Through training the trainers strategies, based on the Skills for change package, and establishing solid coordination between PHC Centres and specialized alcohol units, the program allows general practitioners and nurses to handle with both, risky drinkers and alcoholics.

Key words: Primary health care, brief intervention, risky drinkers, harm reduction, alcoholism. 


\section{INTRODUCCIÓN}

$\mathbf{L}$ a Organización Mundial de la Salud inició en 1981 una estrategia global encaminada a potenciar intervenciones precoces en todos los ámbitos de la salud, enfatizando el papel protagonista de la Atención Primaria en esta labor debido a su amplia cobertura y credibilidad, que convierten a estos equipos en centros estratégicos. Parece evidente que el diagnóstico y la intervención precoz facilitan mejores resultados y permiten prevenir o reducir el impacto de trastornos sociales, médicos y psicológicos, al tiempo que se reducen los costes.

En materia de alcohol, la OMS revisó la clasificación diagnóstica, introduciendo los conceptos de consumo de riesgo y consumo nocivo, junto al ya existente de dependencia del alcohol'. A partir de ahí el consumo de riesgo queda definido como toda pauta de consumo que aumenta el riesgo de sufrir, en el futuro, daños físicos, psíquicos y/o sociales, ausentes en el presente. La clave está en esta ausencia actual de daños o trastornos relacionados que dificultan la detección de este tipo de consumos. Aunque el grado de riesgo no está únicamente relacionado con la cantidad de alcohol ingerida, existe un cierto consenso ${ }^{2}$ en aceptar los límites de 4 unidades de bebida estándar (UBE) diarias en el sexo masculino y 2,5 para las mujeres (En España $1 \mathrm{UBE}=10 \mathrm{gr}$. de alcohol puro ${ }^{3}$ ).

Existe una amplia evidencia acerca de los daños relacionados con el consumo de alcoholl-7. $\mathrm{Y}$ aunque hay una cierta tendencia a creer que sólo las personas con una dependencia alcohólica son susceptibles de sufrir las consecuencias negativas, numerosos estudios evidencian que la mayoría de los daños y trastornos relacionados con el alcohol, incluida la mortalidad, se producen en sujetos consumidores no dependientes ${ }^{8}$.

En este sentido, el Departamento de Sanidad de Estados Unidos advierte que el 38\% de las muertes por accidente de tráfico están relacionadas con el consumo de alcohol ${ }^{9}$. Lo mismo sucede con el $23 \%$ de los accidentes en Francia (frente a un 10\%, por ejemplo, que se relacionan con la fatiga). Estos accidentes relacionados con el alcohol tienden a ser fatales durante la noche y de madrugada ${ }^{10}$.

La American Medical Association advierte que cualquier consumo de alcohol, por mínimo que sea, posee un efecto negativo sobre la percepción, las habilidades, el estilo de conducción y supone un riesgo significativo para la salud y la seguridad y propone una serie de medidas para su control ${ }^{11}$. Del mismo modo advierte que está estrechamente relacionado con las conductas auto y heteroagresivas en la población juvenil (tales como suicidios, homicidios, asaltos sexuales y conductas sexuales de riesgo) ${ }^{12}$.

La OMS analiza detalladamente las consecuencias sociales del consumo de alcohol en un monográfico sobre el tema ${ }^{13}$ : provoca distorsiones en las interacciones sociales (amigos, familia, etc.) tales como inestabilidad emocional, aumento de la agresividad, y por extensión de la criminalidad y el vandalismo, relaciones sexuales no deseadas, violencia doméstica, negligencia familiar, inconsistencia en la autoridad paterna, abandono familiar o ausencia de relaciones familiares, disminución de la productividad laboral y conflictividad en las relaciones laborales, pobre desarrollo escolar en los hijos de bebedores excesivos, etc. Recomienda tomar medidas para incrementar el conocimiento público y la sensibilización acerca de los problemas de salud, seguridad y orden público relacionados con el alcohol. El monográfico finaliza señalando que el coste social del consumo de alcohol representa el $3 \%$ del producto doméstico bruto: en la Unión Europea el coste en el año 1998 fue de entre 65 y 195 millones de dólares, de los cuales el $20 \%$ fueron gastos correspondientes a los servicios médicos, sociales y judiciales.

El balance no es más alentador en España: el $10-15 \%$ de las urgencias y el $4 \%$ de los ingresos hospitalarios están directamente relacionados con el consumo de alcohol. En cuanto a la accidentalidad, entre el 30 y el $50 \%$ de los accidentes mortales de tráfico, el $15-35 \%$ de los accidentes graves de tráfico y 
el $17 \%$ de los accidentes laborales están asociados al consumo de alcohol con un balance final de 19.900 muertos en el año 1997. El coste económico global que el consumo de alcohol genera en nuestro país es de 637.717 millones de pesetas al año ${ }^{14}$. Junto a esto cabe señalar que el 9,5\% de la población general española pueden ser considerados consumidores de riesgo de alcohol ${ }^{15}$.

Parece claro que la detección e intervención precoces son una necesidad tanto para el paciente como para el profesional de la salud, y que el enfoque que debe predominar es el de evitar daños y procedimientos terapéuticos costosos y complejos (tanto económica como técnicamente). De este modo, la intervención en el caso de los individuos que consumen alcohol de forma arriesgada se convierte en una estrategia esencial.

\section{ESTUDIO INTERNACIONAL SOBRE INTER- VENCIÓN PRECOZ EN MATERIA DE ALCO- HOL DESDE LA ATENCIÓN PRIMARIA DE SALUD ${ }^{16}$}

Por este motivo, la OMS puso en marcha, en 1983, el WHO Collaborative Study on Alcohol Early Interventions in Primary Health Care Settings, un estudio multicéntrico internacional centrado en la detección precoz y en el desarrollo de estrategias de intervención apropiadas.

El objetivo final del proyecto es la disminución de la incidencia de los trastornos relacionados con el alcohol y de la incidencia del síndrome de dependencia. Para ello era necesario elaborar un programa simple y eficaz que facilitara a los equipos de Atención Primaria las tareas de detección e intervención precoz en problemas de alcohol. Dicho programa debía constar lógicamente de un instrumento de cribaje eficaz incluso en estadios muy precoces de consumo, y de un protocolo de intervención para aquellas situaciones en que se obtenga un resultado positivo en la exploración. Con esta finalidad, la OMS reunió un equipo de investigadores de dife- rentes países y estructuró un proyecto en 4 fases, con distintos objetivos específicos:

- Fase I: Desarrollo y validación de un cuestionario eficaz (AUDIT).

- Fase II: Evaluación de las intervenciones breves.

- Fase III: Evaluación de las estrategias de marketing y soporte necesarias para la implementación de las intervenciones precoces en la Atención Primaria.

- Fase IV: Diseminación e implementación general de la intervención precoz en materia de alcohol en la Atención Primaria.

\section{Fase I:}

Los consumidores en estadios muy precoces (consumidores de riesgo) difícilmente pueden ser detectados puesto que no presentan signos ni síntomas de problemática asociada al alcohol, no suelen hablar espontáneamente de su consumo y los cuestionarios existentes (CAGE, MAST, MALT, etc.) no son válidos para estos casos, dado que exploran básicamente criterios de dependencia y presentan unos indicadores de validez inaceptables en la detección de consumidores de riesgo. En consecuencia, el esfuerzo inicial se dirigió a construir y validar un cuestionario breve, sencillo y eficaz que recibió el nombre de AUDIT (Alcohol Use Disorders Identification Test) y que presentó una sensibilidad de $93 \%$ y una especificidad de $82 \%{ }^{17-18}$. El cuestionario, que fue pensado para ser autoadministrado, consta de 10 preguntas sencillas que exploran cantidad y frecuencia de consumo, presencia de consecuencias negativas y criterios de dependencia; y permite discriminar consumos de riesgo, consumos nocivos y presencia de dependencia ${ }^{19}$.

EI AUDIT fue validado posteriormente en diversos países, siguiendo la recomendación de la propia OMS, con resultados positivos ${ }^{20}$ ${ }^{24}$. De este modo, en España se han realizado diversas validaciones y análisis que confirman la utilidad del cuestionario en nuestro contexto ${ }^{25-28}$. Una amplia revisión realizada por Fiellin et al. ${ }^{29}$, que abarca 38 estudios internaciona- 
les publicados entre 1966 y 1998, confirma que entre todos los métodos de cribaje evaluados el AUDIT resultó el más efectivo en la identificación de sujetos con consumo de riesgo de alcohol. Idéntica conclusión que la obtenida por Aertgeerts et al. ${ }^{30}$ en un estudio recientemente publicado.

En estos últimos años se han realizado algunos estudios para validar versiones abreviadas del AUDIT ${ }^{30-35}$, entre las que cabe destacar el Five Shot questionnaire de Seppä31 y el Audit-C de Bush ${ }^{32}$, existente también en versión castellana ${ }^{34}$.

\section{Fase II:}

El segundo paso fue investigar qué tipo de intervención era apropiada y eficaz en estos casos. Todos los estudios previos señalaban en sus conclusiones que el consejo breve contribuye a reducir de forma significativa el consumo de alcohol y la prevalencia de consumidores de riesgo, y que su eficacia aumentaba si se repetía periódicamente y se realizaba un seguimiento ${ }^{36}$.

La OMS obtuvo idénticas conclusiones ${ }^{37-38}$ : un $38 \%$ de los pacientes que recibieron simplemente consejo y un $32 \%$ de los que recibieron intervención breve (consejo más pautas y/o seguimiento) redujeron su consumo. La mayoría de los pacientes que efectivamente disminuyeron su consumo, lo hicieron en un $25 \%$ respecto a las cantidades ingeridas previamente. Existe suficiente evidencia científica que avala estos resultados ${ }^{39}$ y por tanto se concluye que las intervenciones breves son un buen recurso para el abordaje de los consumidores de riesgo y que se adaptan a las características de la Atención Primaria por su brevedad.

Estudios recientes muestran que las intervenciones breves pueden ser efectivas incluso a pesar de la falta de interés de los receptores-usuarios del programa ${ }^{40}$, y que, en la práctica clínica, pueden ayudar a los consumidores de riesgo a reducir los niveles de consumo de alcohol. Así mismo, facilitan la abstinencia en pacientes que siguen tratamiento con fármacos y resaltan la efectividad del asesoramiento y tratamiento en aquellos pacientes que no responden a las intervenciones breves solas ${ }^{41}$.

Algunos trabajos muestran además que los folletos de autoayuda aumentan la autoconfianza de los pacientes en el manejo de enfermedades menores y pueden influir en un descenso del número de consultas ${ }^{42}$, aunque este último punto no parece quedar contrastado por todos los estudios ${ }^{43}$.

Todos los datos disponibles sugieren que a largo plazo las intervenciones breves podrían reducir la utilización de los servicios sanitarios $^{44}$. Aunque es necesario más seguimiento y estudios para verificar esta afirmación, lo que sí se ha evidenciado ya es que las intervenciones breves reducen significativamente los costes respecto a otras intervenciones más estructuradas y complejas ${ }^{45}$ (Ver el capítulo 'Efectividad de las técnicas de consejo breve', para una descripción pormenorizada).

Para el desarrollo de esta fase de la investigación se diseñó el programa Drink Less ${ }^{46}$ que contaba con un instrumento válido para la detección de consumidores, una guía de intervención breve y folletos de autoayuda para reforzar el consejo médico.

\section{Fase III:}

Una vez diseñado el instrumento de detección precoz (AUDIT) y demostrada la eficacia de las intervenciones breves, el estadio 1 de la fase III se proponía evaluar las experiencias y actitudes de los profesionales de atención primaria respecto a las actividades preventivas y de promoción de la salud.

En general, la mayoría de los médicos se sienten legitimados para trabajar con bebedores pero más de la mitad se sienten incapaces de hacerlo y poco motivados ${ }^{47-48}$. En Inglaterra, los resultados revelan que los médicos se muestran positivos respecto a la promoción de hábitos saludables, pero no confían inicialmente demasiado en su efectividad como promotores de cambios en el estilo de vida. A pesar de ello la información y el entrenamiento en intervenciones relacionadas con la reducción del consumo de alcohol aumentan 
la percepción de confianza y efectividad ${ }^{49}$. En España se obtuvieron resultados similares ${ }^{50-51}$.

El Estadio 2 pretendía analizar los obstáculos e incentivos para la implementación de las intervenciones breves. Las principales dificultades registradas por los profesionales sanitarios respecto al cribaje y manejo de los pacientes con consumos de riesgo o problemas fueron la falta de tiempo, de formación y de incentivos; el bajo índice de resultados positivos y la ausencia de estrategias estandarizadas y materiales apropiados asociados al consumo de alcohol ${ }^{50-54}$. Ello evidencia la necesidad de ofrecer programas de formación en conceptos básicos y entrenamiento en el manejo de los programas y las estrategias para la intervención.

Un estudio señala como otro de los obstáculos el hecho de que, con frecuencia, se produce poco intercambio de conocimientos entre los investigadores y los profesionales de la práctica clínica ${ }^{55}$. Por tanto puede ser útil un intercambio de información periódica acerca de la eficacia de las intervenciones breves, como estrategia para la difusión de este tipo de abordaje.

El objetivo siguiente, en el estadio 3, consistía en estudiar las mejores estrategias para la difusión e implementación de las intervenciones breves dirigidas a consumidores de riesgo, como mencionamos anteriormente. Para ello se organizó, en todos los países participantes, un ensayo aleatorizado que pretendía comprobar la efectividad de tres distintas estrategias de marketing (contacto telefónico, contacto personal y contacto por correo) y tres estrategias de soporte (formación y soporte, formación sin soporte, no formación ni soporte). En cada país se utilizó una muestra representativa de médicos de Atención Primaria. Cada uno de ellos fue asignado aleatoriamente a una de las condiciones de marketing y era invitado a recibir y conocer el programa de detección precoz e intervención breve. Todos aquellos médicos que aceptaron este ofrecimiento fueron invitados, seguidamente, a utilizar el programa durante tres meses a modo de experiencia piloto.
Los resultados fueron similares en todos los países participantes, incluido España, y muestran que el enfoque promocional facilita la difusión y que el contacto telefónico es la estrategia con mejor relación coste-efectividad para ello ${ }^{56-61}$.

A su vez, los médicos que recibieron entrenamiento y soporte mostraron mayor tendencia a implementar el programa y realizaron un cumplimiento significativamente mejor que el de los otros dos grupos ${ }^{56,62}$.

\section{Fase IV:}

En 1998 se inicia esta última fase de la investigación de la OMS, cuyo objetivo es conseguir la diseminación de los programas de Detección e Intervención Breve (DIB) a toda la Atención Primaria.

En el proceso de desarrollo de protocolos de intervención para la promoción de la salud es necesario utilizar enfoques que combinen las necesidades de la investigación, del propio desarrollo y de la práctica clínica: las intervenciones deben ser aceptables y apropiadas al contexto de la Atención Primaria, deben poder ser focalizadas pero tener una intensidad y duración suficientes como para que puedan tener algún impacto sobre la población y al mismo tiempo poder ser evaluadas. En resumen deben poder adaptarse a las necesidades y características de la práctica clínica sin que se comprometan los principios teóricos ${ }^{63}$.

De la investigación previa se sigue que para una difusión e implementación efectiva de las intervenciones precoces, en el ámbito de la Atención Primaria, es necesario ofrecer:

- Un programa de detección que cuente con instrumentos breves e incluya estrategias claras y estandarizadas de intervención.

- Formación específica que abarque no sólo conceptos sino también habilidades en el abordaje del alcohol.

- Contacto personal, entrenamiento y soporte continuado en la implementación.

Por ello esta última fase se estructuró en 2 etapas: Una primera etapa de preparación y diseño de la difusión y una etapa en la que se 
realizará la difusión propiamente dicha y se evaluarán los resultados.

Se definieron cuatro elementos fundamentales para la preparación de la difusión definitiva: adaptar los materiales, promover un mayor conocimiento y un cambio en la concepción de los problemas relacionados con el alcohol tanto entre los profesionales como en la población general, establecer alianzas estratégicas que posibiliten el alcance comunitario y generalizado de la difusión y, finalmente, desarrollo y evaluación de estrategias de difusión y de intervención comunitarias ${ }^{64}$.

Nuestro grupo de trabajo ha sido el primero en completar esta primera etapa. Fruto de la misma se ha desarrollado un nuevo programa de DIB, el "Beber menos" ("Beveu Menys" en catalán) $)^{65}$, que incluye una versión adaptada y actualizada del material original, un nuevo cuestionario mucho más breve ${ }^{66}$, protocolos diferenciados de intervención para los distintos tipos de problemas de alcohol y material específico para el abordaje motivacional y de la dependencia alcohólica.

Asimismo, se diseñó un programa específico de formación dirigido a los profesionales de Atención Primaria de Salud ${ }^{67}$ a partir de las directrices y resultados obtenidos en diversos grupos focales realizados con profesionales de la Atención Primaria y especialistas en drogodependencias. Dicho programa abarca conceptos esenciales en materia de alcohol y entrenamiento en el manejo del programa "Beveu menys".

En las líneas siguientes describiremos con mayor detalle el Programa "Beveu menys", que marca las directrices a seguir por todos los países en la Fase IV del estudio de la OMS en su segunda etapa.

\section{PROGRAMA "BEVEU MENYS": DETEC- CIÓN PRECOZ E INTERVENCIÓN BREVE EN CONSUMIDORES DE RIESGO DE ALCOHOL}

\section{Programa de formación}

Esta estrategia es una de las principales innovaciones que Catalunya ha introducido en el diseño general del estudio, y pretende resolver la falta de formación que contribuye a generar resistencias en los profesionales de primaria, y con ello facilitar la implementación.

La estrategia global de formación ha sido desarrollada basándonos en la teoría de las etapas del cambio ${ }^{68}$, las estrategias motivacionales $^{69-70}$ y el manual Skills for change ${ }^{71}$ desarrollado por Pip Mason para la OMS (manual sobre técnicas para promover hábitos de vida saludables en general). El programa de formación resultante incorpora asimismo las sugerencias obtenidas en los grupos focales y en los propios cursos de formación de formadores. De este modo, el proceso de entrenamiento y el diseño del programa final de formación se han ido desarrollando paralelamente.

- Formación de formadores: En Catalunya elegimos como formadores a los profesionales de los Centros de Atención a las Drogodependencias porque poseen los conocimientos necesarios para ello y porque pueden coordinarse fácilmente con los Equipos de Atención Primaria (EAP) de su zona, una vez concluido el período de formación, con el objetivo de proporcionarles soporte técnico y de estimular el mantenimiento de las intervenciones con bebedores.

Por otra parte este sistema de organización permite crear, fortalecer y estrechar las relaciones entre los EAP y los equipos de la Red de Atención a las Drogodependencias, que con frecuencia son muy débiles o inexistentes. La finalidad es aunar esfuerzos hasta ahora dispersos para ganar capacidad de intervención y respuesta a la demanda cada vez más creciente.

La formación de formadores se inició con dos seminarios intensivos de dos días de duración con el objetivo de introducir a los profesionales de drogodependencias en la filosofía del programa Skills for Change. Al mismo tiempo pretendíamos discutir los obstáculos que, tanto por parte de los profesionales de Atención Primaria como por parte de los especialistas, podían dificultar la implementación y contrastar opiniones acerca de posibles modelos de intervención en los EAP. 
En total, 70 profesionales de los centros especializados de la Red de Atención a las Drogodependencias asistieron a estos seminarios de formación de formadores, en el curso de los cuales se concretó el guión y estructura del curso de formación para la APS, que consta de cinco sesiones de una hora de duración, con una dinámica interactiva.

- Formación de la APS: Con todo este trabajo previo e información se elaboró y desarrolló un curso que incluye conceptos básicos y estrategias para el abordaje y manejo de los problemas relacionados con el alcohol. Se desarrolla en 5 sesiones, con el siguiente programa:

- Sesión 1: Alcohol y Atención Primaria de Salud. Presentación del programa "Beveu menys".

- Sesión 2: Técnicas de identificación precoz de los bebedores de riesgo.

- Sesión 3: Técnicas de consejo breve y asesoramiento a los bebedores de riesgo.

- Sesión 4: Diagnóstico y tratamiento del síndrome de dependencia alcohólica.

- Sesión 5: Definición de la línea de trabajo y coordinación con el Equipo de Drogodependencias.

En este curso se enfatiza la necesidad de tener en cuenta la opinión del paciente y detectar el estadio del cambio en el que se encuentra como claves para una adecuada y efectiva intervención. La metodología de trabajo es dinámica e interactiva, como ya mencionamos, de modo que las sesiones contienen simultáneamente espacios teóricos, espacios de discusión y reflexión, y ejercicios prácticos con casos clínicos y juegos de rol.

Este material fue evaluado en cuatro experiencias piloto realizadas en EAP y 10 nuevos seminarios cortos de formación de formadores, consiguiendo resultados muy positivos y una acogida muy favorable.

\section{Programa de Detección Precoz e Interven- ción Breve}

- Materiales: El programa definitivo cuenta con una serie de materiales pensados y adaptados para la detección precoz y la intervención breve:
- Tríptico con las pautas para la implementación del programa

- Cuestionario AUDIT y plantilla de corrección

- Cuestionario ISCA

- Guía de monitorización de consumos

- Cuatro Protocolos de intervención

- Folleto "Veus el que beus?" ("¿Ves lo que bebes?"), dirigido a consumidores de riesgo

- Folleto "Salut sense alcohol" ("Salud sin alcohol"), dirigido a pacientes dependientes del alcohol

- Procedimiento:Una vez recibido el curso básico de abordaje de los problemas de alcohol y el programa de intervención "Beveu menys", y establecida la coordinación con el equipo de especialistas en drogodependencias, el Equipo de Atención Primaria (EAP) estará en condiciones de iniciar la implementación del programa.

Se recomienda el cribaje sistemático de toda la población mayor de 16 años cada dos años. La exploración del consumo de alcohol es especialmente importante entre los colectivos con mayor vulnerabilidad (gestantes, población con antecedentes de alcoholismo familiar, etc.) y aquellos que presentan consumos más elevados (jóvenes, hombres adultos de edad media, etc.). Dicho cribaje puede ser realizado tanto por el médico como por la enfermera del EAP aprovechando la consulta del paciente.

La intervención se estructura en varios pasos:

[1] Explorar los hábitos de consumo utilizando indistinta o complementariamente el cuestionario ISCA y/o el AUDIT

[2] Si se detecta un paciente que presenta un consumo de riesgo o dependencia:

- Consultar la "Guía de intervención en problemas derivados del consumo de alcohol en la APS" (uno de los protocolos de intervención), que ayudará a decidir el tipo de intervención más adecuada

- Asesorar al paciente siguiendo las indicaciones de esta guía y de la 
"Guía de monitorización de consumos".

Es importante ayudarle a conocer cuales son los límites máximos de consumo aceptados por la OMS, compararlos con los suyos, discutir con él la medida más adecuada (reducción del consumo o abstinencia) y trabajar la motivación del paciente.

En este sentido, el consejo breve debe incorporar los principios de la teoría del cambio y del abordaje motivacional: es fundamental, como ya mencionamos, detectar en qué estadio del cambio está el paciente y adaptar la intervención, utilizando estrategias que le permitan ir avanzando en la motivación hacia el cambio.

- Ofrecer al paciente el folleto "Veus el que beus?"o "Salut sense alcohol"

- Registrar la intervención realizada en la Historia Clínica (tanto si el resultado del cribaje es negativo como si es positivo)

[3] Negociar con el paciente un seguimiento periódico de su consumo y de la evolución realizada. Es útil implicar al paciente en todo este proceso y negociar con él tanto el tipo de intervención, como la cantidad y ritmo en la reducción del consumo y el seguimiento.

\section{CONCLUSIONES}

Tal como hemos visto en la revisión inicial, la mayor parte de los daños y transtornos relacionados con el alcohol suelen producirse en consumidores no dependientes, con un elevado coste no sólo socio-sanitario sino también psicológico y personal. Ello evidencia la necesidad de desarrollar programas de detección e intervención precoces y de que los profesionales sanitarios simultaneemos cada vez más nuestro trabajo terapéutico con intervenciones preventivas.
La Atención Primaria de Salud constituye un entorno privilegiado para realizar este tipo de intervenciones de un modo oportunista, debido a la elevada credibilidad y cobertura que poseen sobre la población general. Ello explica que sea precisamente en este contexto donde se desarrollen diversos programas y donde se acumule mayor evidencia científica de la eficacia de las intervenciones breves.

El Programa "Beveu menys" es el resultado del trabajo que esta Dirección General de Drogodependencias y Sida, en colaboración con la OMS, ha desarrollado durante los últimos cinco años en esta dirección.

\section{BIBLIOGRAFÍA}

(1) World Health Organization. International Statistical Classification of Diseases and Related Health Problems. Tenth Revision. Geneva: WHO; 1992. Vol 1. p. 320-4.

(2) World Health Organization. International guide for monitoring alcohol consumption and related harm. Geneva: WHO; 2000. p. 51-55. Disponible en: URL: http://www.who.int/substance abuse/PDFfiles/guidemontr_alcohlconsum.pdf

(3) Rodríguez-Martos A, Gual A, Llopis J. La unidad de bebida estándar como registro simplificado del consumo de bebidas alcohólicas y su determinación en España. Med Clín 1999; 112: 44650.

(4) Anderson P. Alcohol and primary health care. Copenhague: WHO Regional Publications (64); 1996. p. 10-4.

(5) World Health Organization. International guide for monitoring alcohol consumption and related harm. Geneva: WHO; 2000. p.1-12/p.81-3. Disponible en: URL: http://www.who.int/substance_abuse/PDFfiles/guidemontr_alcohlconsum.pdf.

(6) Prada C, Del Río MC, Yañez JL, Alvarez FJ. Mortalidad relacionada con el consumo de alcohol. Gac Sanit 1996; 10(55).

(7) Dawson DA, Archer LD. Relative frequency of heavy drinking and the risk of alcohol dependence. Addiction 1993; 88: 1509-18. 
(8) WHO Expert Committee. Problems related to alcohol consumption. WHO Technical Report Series 650. Geneva: WHO; 1980.

(9) Department of Health And Human Services (US). Centers for Disease Control and Prevention (CDC). Motor-vehicle occupant injury: Strategies for increasing use of child safety seats, increasing use of safety belts, and reducing alcoholimpaired driving: A report on recommendations of the task force on community preventive services. The Morbidity and Mortality Weekly Report (MMWR) Series 2001; 50 (RR-7).

(10) Philip P, Vervialle F, Le Breton P, Taillard J, Horne $\mathrm{J}$ A. Fatigue, alcohol, and serious road crashes in France: factorial study of national data. BMJ 2001; 322: 829-30.

(11) American Medical Association (US). Operating vehicles under the influence of alcohol or other drugs/underage drinking and driving. Chicago: AMA. Disponible en: URL: http://www.amaassn.org/special/aos/alcohol1/policy/drinking. htm.

(12) American Medical Association (Web). Disponible en: URL: http://www.ama-assn.org/special/aos/alcohol1/about1b.htm. Accessed June 5, 2001.

(13) Klingemann H, Gomel G, editores. Mapping the social consequences of alcohol consumption. Dordrecht: Kluwer Academic Publishers; 2001.

(14) Portella E, Ridao M, Carrillo E, Ribas E, Ribó C y Salvat M. El alcohol y su abuso: impacto socioeconómico. Madrid: Ed. Médica Panamericana; 1998.

(15) Observatorio Español sobre Drogas: Informe $n^{\circ}$ 4. Madrid: Ministerio del Interior. Delegación del Gobierno para el Plan Nacional sobre Drogas; 2001. p. 13-67.

(16) Saunders JB, Lee NK. Hazardous alcohol use: its delineation as a subthreshold disorder, and approaches to its diagnosis and management. Comprehensive Psychiatry 2000; 41 (2) Supl 1: 95-103.

(17) Saunders JB, Aasland OG. WHO Collaborative Project on the identification and treatment of persons with harmful alcohol consumption. Report on phase I: development of a screening instrument. Geneve: World Health Organization; 1987.

(18) Saunders JB, Aasland OG, Babor TF, de la Fuente JR, Grant M. Development of the Alcohol Use Disorders Identification Test (AUDIT): WHO Collaborative Project on Early Detection of Persons with Harmful Alcohol ConsumptionII. Addiction 1993; 88: 791-804.

(19) Babor TF, De la Fuente JR, Saunders J, et al. AUDIT The alcohol use disorders identification test: guidelines for use in primary health care. Geneva: Substance Abuse Department, World Health Organization; 1992. Report WHO/PSA/ 92.4.

(20) Bohn MJ, Babor TF, Kranzler HR. The Alcohol Use Disorders Identification Test (AUDIT): validation of a screening instrument for use in medical settings. J Stud Alcohol 1995; 56 (4): 423-32.

(21) Schmidt A, Barry KL, Fleming MF. Detection of Problem Drinkers: The Alcohol Use Disorders Identification Test (AUDIT). Southern Medical Journal 1995; 88 (1): 52-9.

(22) Conigrave KM, Hall WD, Saunders JB. The AUDIT questionnaire: choosing a cutt-off score. Addiction 1995; 90 (10): 1349-56.

(23) Volk RJ, Steinbauer JR, Cantor SB, Holzer CE. The Alcohol Use Disorders Identification Test (AUDIT) as a screen for at-risk drinking in Primary Care patients of different racial/ethnic backgrounds. Addiction 1997; 92 (2): 197-206.

(24) Piccinelli M, Tessari E, Bortolomasi M, Piasere O, Semenzin M, Garzotto N, Tanzella M. Efficacy of The Alcohol Use Disorders Identification Test as a screening tool for hazardous alcohol intake and related disorders in Primary Care: a validity study. $\mathrm{Br}$ Med J 1997; 314 (7078): 420-4.

(25) Martínez Delgado JM. Validación de los cuestionarios breves: AUDIT, CAGE y CBA para la detección precoz del síndrome de dependencia de alcohol en Atención Primaria [tesis doctoral]. Cádiz (Sp): Universidad de Cádiz, Departamento de Neurociencias; 1996.

(26) Rubio Valladolid G, Bermejo Vicedo J, Caballero Sanchez-Serrano MC, Santo-Domingo Carrasco J. Validación de la prueba para la identificación de trastornos por uso de alcohol (AUDIT) en Atención Primaria. Rev Clín Esp 1998;198 (1):11-4.

(27) Contel M, Gual A, Colom J. Test para la identificación de trastornos por uso de alcohol (AUDIT): traducción y validación del AUDIT al catalán y castellano. Adicciones 1999; 11(4): 337-47.

(28) Gómez A, Conde A, Aguiar JA, Santana JM, Jorrín A, Betancor P. Utilidad diagnóstica del cuestionario Alcohol Use Disorders Identifica- 
tion Test (AUDIT) para detectar el consumo de alcohol de riesgo en Atención Primaria. Med Clín 2001; 116: 121-24.

(29) Fiellin DA, Reid MC, O'Connor PG. Screening for alcohol problems in primary care: a systematic review. Arch Intern Med 2000; 160(13): 1977-89.

(30) Aertgeerts B, Buntinx F, Ansoms S, Fevery J. Screening properties of questionnaires and laboratory tests for the detection of alcohol abuse or dependence in a general practice population. Br J Gen Pract 2001; 51: 206-17.

(31) Seppä K, Lepistö J and Sillanaukee P. Five-shot questionnaire on heavy drinking. Alcohol Clin Exp Res 1998; 22(8): 1788-91.

(32) Bush K, Kivlahan DR, McDonell MS, Fihn SD, Bradley KA. The AUDIT Alcohol Consumption Questions (AUDIT-C): an effective brief screening test for problem drinking. Arch Intern Med 1998; 158(14): 1789-95.

(33) Daeppen JB, Yersin B, Landry U, Pécoud A, Decrey $\mathrm{H}$. Reliability and validity of the Alcohol Use Disorders Identification Test (AUDIT) imbedded within a General Health Risk Screening Questionnaire: Results of a survey in 332 primary care patients. Alcohol Clin Exp Res 2000; 24 (5): 659-65.

(34) Gual A, Segura L, Contel M, Heather N, Colom J. AUDIT-3 and AUDIT-4: effectiveness of two short forms of the Alcohol Use Disorders Identification Test (AUDIT). Alcohol Alcohol. Submitted 2001.

(35) Gordon AJ, Maisto SA, McNeil M, et al. Three questions can detect hazardous drinkers. J Fam Pract 2001; 50(4):313-20.

(36) Wallace P, Cutler S, Haines A. Randomized controlled trial of general practitioner intervention in patients with excessive alcohol consumption. BMJ 1988; 297: 663-68.

(37) WHO Brief Intervention Study Group. A crossnational trial of brief intervention with heavy drinkers. Am J Pub Health 1996; 86: 948-955.

(38) Babor TF, Grant M. WHO Collaborating investigators project on identification and management of alcohol-related problems. Combines analyses of outcome data: the cross-national generalizability of brief interventions. Report on phase II: a randomized clinical trial of brief interventions in primary health care. Copenhagen: WHO; 1992.
(39) Nuffield Institute for Health (UK). Brief interventions and alcohol use. Effective Health Care Bulletin 1993; (7).

(40) Kishchuk N, Peters C, Towers AM, Sylvestre M, Bourgault C, Richard L. Formative and effectiveness evaluation of a worksite program promoting healthy alcohol consumption. Am J Health Promot 1994; 8(5): 353-62.

(41) Fleming M, Manwell LB. Brief intervention in primary care settings. A primary treatment method for at-risk, problem, and dependent drinkers. Alcohol Res Health 1999; 23(2): 128-37.

(42) Little P, Somerville J, Williamson Y, Warner G, Moore M, Wiles R, et al. Randomised controlled trial of self management leaflets and booklets for minor illness provided by post. BMJ 2001; 322: 1214-7.

(43) Heaney D, Wyke S, Wilson P, Elton R, Rutledge P. Assessment of impact of information booklets on use of healthcare services: randomised controlled trial. BMJ 2001; 322: 1218-21.

(44) Freeborn DK, Polen MR, Hollis JF, Senft RA. Screening and brief intervention for hazardous drinking in an HMO: effects on medical care utilization. J Behav Health Serv Res 2000; 27(4): 446-53.

(45) Wutzke SE, Shiell A, Gomel MK, Conigrave KM. Cost effectiveness of brief interventions for reducing alcohol consumption. Soc Sci Med 2001; 52: 863-70.

(46) University of Sidney (Au). Drink-less. Sidney: The University; 1994.

(47) Anderson P. Managing alcohol problems in general practice. Br Med J 1985; 290: 1873-75.

(48) Anderson P, Clement C. The AAPPQ revisited. The management of general practitioners'attitudes to alcohol problems. British Journal of Addiction 1980; 75: 413-31.

(49) McAvoy BR, Kaner EF, Lock CA, Heather N, Gilvarry E. Our Healthier Nation: are general practitioners willing and able to deliver? A survey of attitudes to and involvement in health promotion and lifestyle counselling. $\mathrm{Br} J$ Gen Pract 1999; 49(440):187-90.

(50) Contel M. Actitud de los profesionales de Atención Primaria respecto al abordaje del alcohol. Adicciones 1999. Libro de resúmenes XXVI Jornadas Nacionales de Socidrogalcohol; 1992 Mar; Tenerife, España.

(51) Contel M, Gual A, Colom J. Professional's attitude as a factor of prevention. Proceedings of 
the 4th ERIT European Conference; 2000 Feb; Porto, Portugal.

(52) Gomel MK, Saunders JB, Burns L, Hardcastle DM, Sumich M. Dissemination of early intervention for harmful alcohol consumption in general practice. Health Promotion Journal of Australia 1994; 4(2): 65-9.

(53) Owens L, Gilmore IT, Pirmohamed M. General practice nurses' knowledge of alcohol use and misuse: a questionnaire survey. Alcohol 2000;35(3):259-62.

(54) Aalto M, Pekuri P, Seppä K. Primary health care nurses' and physicians' attitudes, knowledge and beliefs regarding brief intervention for heavy drinkers. Addiction 2001; 96: 305-11.

(55) Nutbeam D. Improving the fit between research and practice in health promotion: overcoming structural barriers. Can J Public Health 1996;87 Supl 2: S18-23.

(56) Gomel MK, Wutzke SE, Hardcastle DM, Lapsley H, Reznik RB. Cost-effectiveness of strategies to market and train primary health care physicians in brief intervention techniques for hazardous alcohol use. Soc Sci Med 1998;47(2): 203-11.

(57) Lock CA, Kaner EF, Heather N, McAvoy BR, Gilvarry $E$. A randomized trial of three marketing strategies to disseminate a screening and brief alcohol intervention programme to general practitioners. Br J Gen Pract 1999; 49(446): 695-8.

(58) Hansen LJ, Olivarius N, Beich A, Barfod S. Encouraging GPs to undertake screening and a brief intervention in order to reduce problem drinking: a randomized controlled trial. Fam Pract 1999;16(6):551-7.

(59) Struzzo P. Prevention of alcohol-related problems. From therapy to primary health care: experience at the Udine "Healthy City". Recenti Prog Med 1999; 90(2): 69-72.

(60) Lock CA, Kaner EF. Use of marketing to disseminate brief alcohol intervention to general practitioners: promoting health care interventions to health promoters. J Eval Clin Pract 2000; 6(4): 345-57.

(61) Colom J. Phase III. Report of Catalonia. Proceedings of the $4^{\text {th }}$ Meeting of the National Counterparts of the European Alcohol Action Plan;
1998 Oct 15-17; Madrid, Spain.

(62) Kaner EF, Lock CA, McAvoy BR, Heather N, Gilvarry $E$. A RCT of three training and support strategies to encourage implementation of screening and brief alcohol intervention by general practitioners. Br J Gen Pract 1999; 49(446): 699-703.

(63) Rogers S, Humphrey C, Nazareth Y, Lister S, Tomlin Z, Haines A. Designing trials of interventions to change professional practice in primary care: lessons from an exploratory study of two change strategies. BMJ 2000;320 (7249):1580-3.

(64) Heather N. Phase IV: implementing countrywide early identification and brief interventions strategies in primary health care. WHO collaborative project on identification and management of alcohol-related problems in primary health care. Copenhage (DK): WHO; 1998 (no publicado).

(65) Direcció General de Drogodependències y Sida. Programa "Beveu Menys". Barcelona (Sp): Departament de Sanitat y Seguretat Social; 2001.

(66) Gual A, Contel M, Segura L, Ribas A, Colom J. El ISCA (Interrogatorio Sistematizado de Consumos Alcohólicos), un nuevo instrumento para la identificación precoz de bebedores de riesgo. Med Clín 2001; 117:685-9.

(67) Colom J, Gual A, Contel M.. Training pack for the implementation of the WHO "Drink-less Programme". Barcelona (Sp): Direcció General de Drogodependències y Sida, Generalitat de Catalunya; 2001.

(68) Prochaska JO, DiClemente CC. Towards a comprehensive model of change. In: Miller WR, Heather N, editores. Treating addictive behaviors: processes of change. New York: Plenum; 1986.

(69) Miller WR, Rollnick S. Motivational interviewing. New York: Guilford Press; 1991.

(70) Rollnick S, Heather N, Bell A. Negotiating behaviour change in medical settings: the development of brief motivational interviewing. J Ment Health 1992; 1:25-37.

(71) World Health Organization. Skills for Change. Copenhagen (DK): WHO Regional Office for Europe; 1998. 